\title{
Prior mating success can affect allocation towards future sexual signaling in crickets
}

Fitness is often correlated with the expression level of a sexually selected trait. However, sexually selected traits are costly to express such that investment in their expression should be optimised to maximize their overall fitness gains. Social interactions, in the form of successful and unsuccessful matings, may offer males one type of feedback allowing them to gauge how to allocate their resources towards sexual signaling. Here we tested whether adult male black field crickets (Teleogryllus commodus) modify the extent of their calling effort (the sexually selected trait) in response to successful and unsuccessful matings with females. To examine the effect that mating interactions with females have on investment into sexual signaling, we monitored male calling effort after maturation and then provided males with a female at two points within their life, manipulating whether or not males were able to successfully mate each time. Our results demonstrate that males alter their investment towards sexual signaling in response to successful matings, but only if the experience occurs early their life. Males that mated early in their life decreased their calling effort sooner than males that were denied a mating. Our results demonstrate that social feedback in the form of successful and unsuccessful matings has the potential to alter the effort a male places in sexual signaling. 
2 Rachel Chiswell ${ }^{1}$, Madeline Girard ${ }^{2}$, Claudia Fricke ${ }^{3}$, and Michael M. Kasumovic ${ }^{1 *}$

3 1. Evolution \& Ecology Research Centre, School of Biological, Earth \& Environmental Sciences,

4 The University of New South Wales, Kensington, Sydney 2052 NSW, Australia

5 2. Department of Environmental Science, Policy and Management, University of California,

6 Berkeley, California, United States of America

7 3. Institute for Evolution and Biodiversity, University of Muenster, Muenster, Germany

8 * Corresponding Author: m.kasumovic@unsw.edu.au 


\section{Introduction}

10 In many organisms, sexually selected traits are costly to produce and their expression often

11 varies as a consequence of resource abundance (i.e. condition dependence; $\underline{\text { Rowe and Houle }}$

12 1996; Cotton et al. 2004) (e.g., morphological traits: Bonduriansky and Rowe 2003; ejaculate

13 traits: Fricke et al. 2008; behavioural traits: Lomborg and Toft 2009). This is most strongly

14 reinforced by studies demonstrating longevity costs of extra investment in sexual signalling

15 (Hunt et al. 2004), although questioned by studies that demonstrate the opposite (Kotiaho et al.

16 1999; Papadopoulos et al. 2004). Despite the well-established link between nutrient/resource

17 abundance and trait expression (Bonduriansky 2007), resources are only one of the many factors

18 that influence the expression of sexually selected traits. Decades of competition research suggests

19 that the social environment is an important contributor that results in plasticity in the expression

20 of sexually selected traits after maturity. For example, in many species of mammals, birds, and

21 invertebrates, dominant individuals are able to moderate the behaviour of subordinate individuals

22 by enforcing a lower level of sexual signalling (Qvarnström 1997; Bekoff and Dugatkin 2000;

23 Tibbetts and Dale 2004).

24 As studies explore the effect of intra- and intersexual social interactions on individual

25 behaviour, a secondary view that is gaining some traction in the literature is that plasticity in

26 sexual signalling is a result of individual decisions resulting from conspecific feedback (i.e.

27 information received from interacting with and eavesdropping on conspecifics) rather than

28 enforcement. For example, studies manipulating the social environment during immaturity

29 demonstrate that males of a wide variety of species alter how they allocate resources towards life-

30 history (Gage 1995; Kasumovic and Andrade 2006; Walling et al. 2007; Kasumovic et al. 2011),

31 metric (Rodd et al. 1997; Kasumovic and Andrade 2006; Kasumovic et al. 2011; DiRenzo et al.

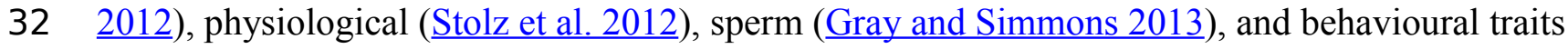


33 (Bailey et al. 2010; Kasumovic et al. 2012b) at maturity. Additionally, adult males show plastic

34 responses when exposed to rivals, enabling them to quickly respond to changes in the

35 environment (Bretman et al. 2011) often with significant fitness benefits (Bretman et al. 2009).

36 The feedback provided from the composition of the social environment can thus play an

37 important role on individual perception of the intensity of competition, and therefore, the 38 expression of sexually selected traits (Kasumovic and Brooks 2011).

39 The idea that changes in sexual signalling are a result of social feedback rather than social 40 enforcement is strengthened by the theoretical and empirical understanding of winner and loser

41 effects in the competition literature. Repeated competitions with rivals for access to resources

42 provides individuals with feedback on their own performance relative to rivals within a

43 population (

44 et al. 2010). Individuals that win more contests are more likely to escalate future contests because

45 they have a favourable perception of their fighting ability, while individuals that lose more

46 contests have a less-favourable perception of their own fighting ability (i.e. winner/loser effects;

47 Hsu et al. 2006). In this sense, individuals are altering their behaviour and the expression of a

48 sexually selected trait not as a consequence of condition, per se, but rather as a result of social

49 interactions that indicate their relative fighting ability.

50 Fawcett and Bleay (2009) theoretically extended the idea of intra-sexual interactions

51 modifying self-perception of fighting ability to inter-sexual interactions altering a male's

52 perception of his own attractiveness. Through their model, they demonstrate that experiences of

53 rejection reduce choosiness and experiences of acceptance increase it, similar to winner and loser

54 effects in direct male-male competitions (e.g., Kasumovic et al. 2009). Empirical evidence of

55 mate rejection and acceptance in zebra finches (Taeniopygia guttata) supports this idea (Pariser et

56 al. 2010; Royle and Pike 2010). Moreover, when Pariser et al. (2010) manipulated individual 
57 attractiveness through coloured leg rings, they found that zebra finch males subsequently changed

58 body mass, condition and courtship display rate, demonstrating the importance of social feedback

59 in trait maintenance. These results illustrate that artificial ornamentation not only manipulated the

60 female's perception of the male but also changed the male's perception of his own attractiveness,

61 via the behavioural 'mirror' of female feedback. Whether the change in courtship rates (i.e.,

62 sexual signalling effort) was a result of direct social feedback or a consequence of reduced mass

63 and condition, however, is difficult to determine.

64 Our goal in this study was to examine whether feedback from females in the form of

65 successful or unsuccessful matings alters future male investment in sexually selected signals. We

66 used the Australian black field cricket (Teleogryllus commodus) to investigate this question as

67 males call throughout their adult lifetime in an attempt to attract mates. Males that call more

68 attract significantly more mates (Bentsen et al. 2006), but calling is likely energetically costly

69 (e.g.. Hoback and Wagner Jr. 1997 on Gryllus lineaticeps) as it leads to a shorter lifespan (Hunt et

70 al. 2004; Hunt et al. 2006); thereby demonstrating the trade-off between reproductive effort and

71 lifespan. In addition, there is evidence in this and other closely related species that social

72 interactions and feedback affect juvenile developmental tactics (Bailey et al. 2010; Kasumovic et

73 al. 2011; DiRenzo et al. 2012; Kasumovic et al. 2012b), adult male behaviour (

74 and lifetime calling effort (Zajitschek et al. 2012; Callandera et al. 2013). As a result, we

75 predicted that males would differentially alter investment towards sexual signalling as a function

76 of successful mating experiences. We also wanted to explore whether we could partition the

77 effects of mating itself and interactions with females to better understand how males invest in

78 sexual signalling.

79 We hypothesized that successful mating opportunities would result in positive feedback that

80 would reinforce the current signalling effort as being sufficient to attract and successfully mate 
81 with females. In contrast, we hypothesized that unsuccessful mating opportunities would provide

82 negative feedback on reproductive effort and further suggest a choosier mating environment

83 which should result in males increasing their calling effort. Since calling effort is costly, we

84 predicted that these changes in calling effort should have subsequent effects on lifespan.

\section{Methods}

86 The crickets used for this experiment were first generation descendants of approximately 300

87 immature individuals collected at Smith's Lake, New South Wales (32 $\left.22^{\prime} \mathrm{S}, 1^{\circ} 2^{\circ} 30^{\prime} \mathrm{E}\right)$ in 2012.

88 We removed penultimate male and female crickets from the common population and reared them

89 individually in a plastic container $(5 \times 5 \times 3 \mathrm{~cm})$ with an egg carton for shelter, and ad libitum food

90 (Friskies Go Cat Senior) and water. We kept all the individuals in a controlled temperature room

91 of $28^{\circ} \mathrm{C}$ on a $14: 10$ light to dark cycle and replaced their water and food weekly. We checked

92 crickets daily for adult eclosion and within 24 hours of eclosing, we weighed individuals using an

93 electronic balance and measured pronotum width as a measurement of size. Adult females

94 remained in individual containers within a controlled temperature room until required for

95 matings.

96 Upon maturity, we placed males in a custom-built electronic monitoring device (callbox; see

97 Lailvaux et al. 2010) overnight each day to determine daily calling effort. Males were kept in

98 individual containers $(5 \times 5 \times 3 \mathrm{~cm})$, which were then placed in plastic containers $(14 \times 6 \times 6 \mathrm{~cm})$

99 surrounded by acoustic foam to keep males in acoustic isolation. We monitored male calling over

100 a 12 hour period each night and used a male's time spent calling each night in seconds as our

101 estimate of calling effort. A male's placement within the callbox was randomized every other day.

102 Although males were placed within the callbox upon maturity, we only monitored male

103 calling effort from age 7-28. On day 14 and 21, we removed males from their individual cages 
104 and placed them in a $10 \times 5 \times 4 \mathrm{~cm}$ mating arena. As males generally begin calling between days 5 -

10510 , and usually reach their peak calling effort around day 20-22, we chose day 14 and 21 to

106 examine how social interactions affect calling effort after males have begun calling to before

107 reaching their peak. We chose two mating opportunities as males in a natural population of

108 another species of cricket (Gryllus campestris) averaged two matings throughout their lifetime

109 (Rodriguez-Munoz et al. 2010) and we wanted to examine whether single mating opportunities

110 affected age-specific calling effort. Once in the mating enclosure, a virgin female was placed

111 within the container for 24 hours along with her water and food containers and males were either

112 allowed to interact and mate with the female or allowed to interact but denied the opportunity to

113 mate. Previous studies demonstrate that males always mate when placed with a female over 24

114 hours (

115 were denied access to females, we placed females inside an overturned $100 \mathrm{ml}$ transparent plastic

116 cup. Each cup had approximately twenty holes evenly distributed throughout the cup and no

117 bigger than $4 \mathrm{~mm}$ in diameter. These holes were large enough to allow males to pass the front of

118 their head and antenna through, but not their body. These holes thus allowed pheromonal,

119 acoustic and visual communication to occur between the males and females, but prevented any

120 matings from occurring.

121 As males were either granted or denied a mating at each mating opportunity (day 14 and 21),

122 we had a $2 \times 2$ design which resulted total of four experimental treatments: (i) an 'early mating'

123 treatment where males were granted a mating on day 14 but denied a mating on day 21 , (ii) a

124 'late mating' treatment where males were granted a mating on day 21 but denied a mating on day

125 14, (iii) a 'multiple mating' treatment where males were granted a mating on both day 14 and 21,

126 and (iv) a 'denied mating treatment' where males are prevented from mating on both day 14 and

127 21. We also had an isolation (control) treatment where males were placed in a mating arena but 
128 no females were introduced. Since no female was introduced, we placed the male's own egg

129 carton and water bottle in the mating arena to ensure he had food and water. All the males were

130 returned to the call box the day after their mating treatment and calling effort was monitored until

131 day 28. After 28 days, we removed males from the call box and monitored their survival.

132 Although all previous studies demonstrate that male $T$. commodus mate immediately with

133 females when they are available (Hall et al. 2008), there is the possibility that some males did not

134 mate or that some males mated more often than others. This, however, would add noise to the

135 differences in age-specific calling effort between treatments making it less likely to see a

136 significant effect of a mating rather than skewing it towards significance.

\section{Statistical analysis}

138 In all our examinations, we used a mixed-model approach with individual identity as a

139 random factor using the nlme package (Pinheiro et al. 2009) in R 3.1.0 (R Core Team, 2014). We

$140 \log$ transformed calling effort and used the orthogonal quadratics to avoid issues with

141 multicollinearity. Since males began calling at different ages resulting in different rates of calling

142 increase early in their lifetime, it resulted in significantly different early portions of the calling

143 curve. As a result, we limited our analysis to calling effort between days 15 (the first mating) and

14428 to ensure that calling effort prior to the treatment did not skew our results and that we focused

145 on changes in calling effort as a function of the treatment.

146 As the individuals in the isolation treatment never experienced any females throughout their

147 lifetime, there was no direct way to examine whether males in the isolation treatment behaved

148 differently to all the mating treatments without classifying each treatment separately, thus

149 decreasing our power. As such, we first examined whether age-specific calling effort of males in

150 the isolation treatment differed from the males in the denied mating treatment by adding a 


\section{PeerJ Reviewing Manuscript}

151 treatment effect to a model containing only the linear and quadratic components of age. Since

152 adding treatment to the model did not improve our fit (see results), we decided to use the

153 isolation treatment by combining it with the denied treatment in our further analyses to maximize

154 our sample size and power.

155 To examine whether early and late matings each individually or jointly affected age-specific

156 calling effort, we coded each mating (early and late) as a function of being granted or denied.

157 Following Zajitschek et al. (2012), we began with a full model consisting of the two-way

158 interactions between early and late mating crossed with the linear and quadratic effects of age as

159 well as all the lower order terms involved in this interaction. We then used the model selection

160 function to reduce the model using the Akaike Information Criteria (AIC) (Akaike 1983) with

161 models that decrease the AIC by at least 2 providing a significantly better fit (Burnham and

162 Anderson 2002). We initially used maximum likelihood for model selection and then report

163 statistics using REML. We visualized male age-specific calling effort using non-parametric

164 splines generated with the general additive mixed model package (gamm4, Wood 2009).

165 We used an ANOVA to examine whether total calling effort (log transformed) differed as a

166 consequence of either an early or late successful mating, and a GLM to examine whether lifespan

167 was affected by a successful early or late mating with total calling effort as a covariate. These

168 were performed in R 3.1.0.

\section{Results}

170 A total of 102 males were approximately equally distributed within each of the five

171 treatments (Early: 22, Late: 22, Multiple: 19, Denied: 20, Isolation: 19). Each treatment began

172 with 25 individuals and the difference in the sample size is a result of individuals that didn't call

173 at all within the first 14 days of their life being removed from the experiment. We removed these 
174 individuals since males that do not call by this point also did not call throughout the rest of their

175 life, likely because they were following a different mating strategy (i.e. sneaking rather than

176 calling; Bailey et al. 2010). As a result, keeping these non-calling males would skew results in the

177 different treatments. In addition, although individuals may alter other behaviour, we cannot study

178 changes in calling effort if males do not call. No individuals died before 28 days. As one

179 individual escaped from the Late mating treatment prior to death, the lifespan analysis consists of

180101 individuals.

181 In our initial comparison of the age-specific calling curves between individuals in the

182 isolation and denied treatment, the addition of treatment in the model did not significantly

183 improve the fit $(\log$-likelihood difference $=0.66, \mathrm{P}=0.42)$. As a result, we combined the two

184 treatments to improve our sample size.

185 The model that best described the variation in age-specific calling effort included the early

186 mating term, the linear and quadratic effects of age, as well as the interaction between the early

187 mating term and the linear and quadratic effects of age (Table 1). This results in changes in the

188 curvature of lifetime male calling effort with age (Fig. 1). Only the quadratic terms were

189 significant, suggesting that it is the non-linear component that is affected with an early mating.

190 This is supported by the significant effect of the quadratic term for males that mated early, while

191 there was no significant effect of the quadratic term for males that were denied the early mating

192 (Table 2).

193 There was no difference in total calling effort as a function of a successful early $\left(F_{1,99}=0.08\right.$, $194 P=0.78)$ or late $\left(F_{1,99}=0.00, P=0.99\right)$ mating between treatments. Individual lifespan also was not 195 affected by either a successful early $\left(t_{1,97}=-0.0 .5, P=0.96\right)$ or late $\left(t_{1,97}=0.82, P=0.41\right)$ mating, or 196 total calling effort $\left(t_{1,97}=0.95, P=0.34\right)$. 
197 Discussion

198 Given the strong selection for high rates of acoustic calling in Teleogryllus commodus

199 (Bentsen et al. 2006), there is likely to be an evolutionary benefit for males to optimise calling

200 effort over their lifespan. Both diet (Hunt et al. 2004; Judge et al. 2008; Bertram et al. 2011) and

201 the social environment encountered prior to maturity (Kasumovic et al. 2012a; Kasumovic et al.

$202 \underline{2012 b})$ contribute to the variance in male age-specific calling effort trajectories. Here we

203 investigated whether variation in age-specific calling effort may also be a response to social

204 feedback in the form of successful and unsuccessful matings. Our results suggest that social

205 feedback from matings occurring early in a male's lifetime have the potential to alter how they

206 invest in sexual signalling throughout their life (Fig. 1). Despite this shift in the trajectory of

207 calling effort, there was no difference in the total calling effort of males in the different

208 treatments and no effect on male lifespan.

209 A previous study examining the effect of diet and weekly successful matings in T. commodus

210 found that males that were mated on a weekly basis had a lower lifetime calling effort than virgin

211 males (Zajitschek et al. 2012). Our results broaden our understanding of sexual investment by

212 males as we demonstrate that a shift in investment can occur as a consequence of a single

213 successful mating. Our mating treatments were positioned such that they occur before a virgin

214 male's peak calling effort in an attempt to understand whether mating interactions affect how

215 males reach their peak calling effort. We show that an unsuccessful mating prior to a male

216 reaching his peak calling effort results in males maintaining their calling effort for a longer period

217 of time relative to individuals that are successful early in their lifetime (Fig. 1). Our results thus

218 provide evidence that males use social feedback from females to alter their investment towards

219 sexual signalling as seen in other species (Pariser et al. 2010; Royle and Pike 2010; e.g.,

220 Callandera et al. 2013). Most importantly, we demonstrate that males that mate early in their 
221 lifetime subsequently differ in their signalling effort compared to males that do not mate early. As

222 a result, no social feedback from conspecific males in the form of social enforcement is necessary

223 (Callandera et al. 2013).

224 Another possibility is, however, that mating itself was costly enough that it decreased male

225 investment in sexual signalling. For example, in tree crickets (Oecanthus nigricornis), males

226 provide a nuptial gift and what were considered high quality males decreased their investment in

227 subsequent gifts in response to perceived female density (Bussière et al. 2005). Although $T$.

228 commodus do not produce gifts as large as O. nigricornis, the spermatophores produced prior to

229 courtship may be costly enough to result in a decrease in calling effort. Our mating design allows

230 us to further dissect this possibility. First, regardless of whether males successfully mated or not,

231 all male T. commodus perform the costly courtship calling (Hack 1998) prior to mating and

232 produce a spermatophore in preparation for mating (Loher 1974). This coupled with the fact that

233 there was no difference in lifespan as a function of a successful mating suggests that similar

234 contact costs were paid by all males in the mating treatments. Second, we did not see a change in

235 age-specific calling effort as a function of a granted or denied second mating, further suggesting

236 that decreased calling effort is not a consequence of a decreased energetic pool. Finally, males in

237 the isolation (control) treatment where the extra-costly courtship calling and spermatophore

238 transfer was presumably not triggered did not significantly differ in age-specific calling effort

239 from males in the denied treatment. These factors suggest that a change in age-specific calling

240 effort is a function of female feedback rather than spermatophore or mating costs, but further

241 studies are necessary to rule this possibility out completely.

242 Despite the differences in age-specific calling effort between treatments, we saw no

243 difference in lifetime calling effort or male lifespan between males as a consequence of

244 successful matings in either the early or late period. This is in contrast to previous results 
245 demonstrating that repeated matings reduce calling effort (Zajitschek et al. 2012). This difference

246 may be because males were continually mated throughout their life, rather than provided with

247 only two matings well-spaced through the early period of their life in our experiment.

248 Nevertheless, it may be beneficial for males to alter their signalling effort in response to female

249 feedback early in their life because a male's likelihood of survival under field conditions is

250 significantly lower than a female's (15.2 versus 21.4 days, respectively) (Zajitschek et al. 2009).

251 Males that decrease their calling effort in response to their success may increase their natural

252 lifespan by decreasing their mortality hazard rate from parasites and predators that exploit sexual

253 signals ( Zuk and Kolluru 1998). This idea, however, requires further testing in a field

254 scenario.Our results add to the growing list of studies demonstrating that social feedback can

255 influence individual reproductive effort in a myriad of ways ranging from experiencing them

256 prior to maturity (Kasumovic and Brooks 2011), to direct (Fawcett and Johnstone 2003; $\underline{\text { Hsu et }}$

257 al. 2006) and indirect (Callandera et al. 2013) intrasexual feedback, and direct intersexual

258 feedback (Fawcett and Bleay 2009; Pariser et al. 2010; Royle and Pike 2010). The experience an

259 individual gains as a function of conspecific interactions is thus an important determinant of the

260 level of trait expression, selection and therefore, how researchers view the concept of individual

261 quality (Lailvaux and Kasumovic 2011). Our results, in combination with others, highlight the

262 importance of examining and considering the social environment and social experience when

263 exploring male mating strategies and investment towards sexual signalling.

\section{Acknowledgements}

265 Thanks to Heather Try for help in rearing crickets, Rob Brooks for looking at earlier drafts in the 266 manuscript, and a huge shout out to Matt Hall and James Smith for discussions about statistics.

267 We would also like to thank Nathan Bailey, two other anonymous reviewers and especially Luc

268 Bussière, for helpful comments during the review process. MBG was supported by NSF's East 


\section{PeerJ Reviewing Manuscript}

269

270

271

Asia and Pacific Summer Institue program. CF was supported by travel grants from Helge Ax:son

Johnsons Stiftelse and Stiftelse Lars Hiertas Minne. MMK was supported by an Australian

Research Council DECRA Fellowship.

272

273

274

275

276

277

278

279

280

281

282

283

284

285

286

287

288

289

290

291

292

293

294

295

296

297

298

299

300

301

302

303

304

305

306

307

308

309

310

311

312

\section{References}

Akaike H (1983) Information measures and model selection. Bulletin of the International Statistical Institute 44:277-291

Bailey NW, Gray B, Zuk M (2010) Acoustic experience shapes alternative mating tactics and reproductive investment in male field crickets. Curr Biol 20:845849

Bekoff M, Dugatkin LA (2000) Winner and loser effects and the development of dominance relationships in young coyotes: an integration of data and theory. Evol Ecol Res 2:871-883

Bentsen CL, Hunt J, Jennions MD, Brooks R (2006) Complex multivariate sexual selection on male acoustic signaling in a wild population of Teleogryllus commodus. Am Nat 167:E102-E116

Bertram SM, Thomson IR, Auguste B, Dawson JW, Darveau CA (2011) Variation in cricket acoustic mate attraction signalling explained by body morphology and metabolic differences. Anim Behav 82:1255-1261

Bonduriansky R (2007) The evolution of condition-dependent sexual dimorphism. Am Nat 169:9-19

Bonduriansky R, Rowe L (2003) Interactions among mechanisms of sexual selection on male body size and head shape in a sexually dimorphic fly. Evolution 57:2046-2053

Bretman A, Fricke C, Chapman T (2009) Plastic responses of male Drosophila melanogaster to the level of sperm competition increase in male reproductive fitness. Proceedings B: Biological Sciences 276:1705-1711

Bretman A, Gage MJG, Chapman T (2011) Quick-change artists: male plastic behavioural responses to rivals. Trends in Ecology \& Evolution 26:467-473

Burnham KP, Anderson DR (2002) Model selection and multi-model inference, 2nd ed. edn. Springer, New York, NY.

Bussière LF, Basit HA, Gwynne DT (2005) Preferred males are not always good providers: female choice and male investment in tree crickets. Behav Ecol 16:223-231

Callandera S, Kahna AT, Hunt J, Backwell PRY, Jennions MD (2013) The effect of competitors on calling effort and life span in male field crickets. Behav Ecol:doi: 10.1093/beheco/art059

Cotton S, Fowler K, Pomiankowski A (2004) Do sexual ornaments demonstrate heightened condition-dependent expression as predicted by the handicap hypothesis? Proc Roy Soc London Ser B 271:771-783

DiRenzo N, Pruitt JN, Hedrick AV (2012) Juvenile exposure to acoustic sexual signals from conspecifics alters growth trajectory and an adult personality trait. Anim Behav:http://dx.doi.org/10.1016/j.anbehav.2012.07.007

Fawcett TW, Bleay C (2009) Previous experiences shape adaptive mate preferences. Behav Ecol 20:68-78 
313

314

315

316

317

318

319

320

321

322

323

324

325

326

327

328

329

330

331

332

333

334

335

336

337

338

339

340

341

342

343

344

345

346

347

348

349

350

351

352

353

354

355

356

357

358

359

360

361

362

363

364

365
Fawcett TW, Johnstone RA (2003) Mate choice in the face of costly competition. Behav Ecol 14:771-779

Fawcett TW, Johnstone RA (2010) Learning your own strength: winner and loser effects should change with age and experience. Proceedings B: Biological Sciences 277:1427-1434

Fricke C, Bretman A, Chapman T (2008) Adult male nutrition and reproductive success in Drosophila melanogaster. Evolution 62:3170-3177

Fuxjager MJ, Marler CA (2010) How and why the winner effect forms: Influences of contest environment and species differences. Behavioral Ecology 21:37-45

Gage MJG (1995) Continuous variation in reproductive strategy as an adaptive response to population density in the moth Plodia interpunctella. Proc R Soc Lond B 261:25-30

Gray B, Simmons LW (2013) Acoustic cues alter perceived sperm competition risk in the field cricket Teleogryllus oceanicus. Behavioral Ecology 24:982-986

Hack MA (1998) The energetics of male mating strategies in field crickets (Orthoptera: Gryllinae: Gryllidae). J Insect Behav 11:853-867

Hall MD, Bussière LF, Hunt J, Brooks R (2008) Experimental evidence that sexual conflict influences the opportunity, form and intensity of sexual selection. Evolution 62:2305-2315

Hall MD, Lailvaux SP, Blows MW, Brooks R (2010) Sexual conflict and the maintenance of multivariate genetic variation. Evolution 64:1697-1703

Hoback WW, Wagner Jr. WE (1997) The energetic cost of calling in the variable field cricket, Gryllus lineaticeps Physiol Entomol 22:286-290

Hsu Y, Earley RL, Wolf LL (2006) Modulation of aggressive behaviour by fighting experience: mechanisms and contest outcomes. Biol Rev 81:33-74

Hunt J, Brooks R, Jennions MD, Smith MJ, Bentsen CL, Bussière LF (2004) High-quality male field crickets invest heavily in sexual display but die young. Nature 432:1024-1027

Hunt J, Jennions MD, Spyrou N, Brooks R (2006) Artificial selection on male longevity influences age-dependent reproductive effort in the black field cricket Teleogryllus commodus. Am Nat 168:E72-86

Judge KA, Ting JJ, Gwynne DT (2008) Condition dependence of male life span and calling effort in a field cricket. Evolution 62:868-878

Judge KA, Ting JJ, Schneider J, Fitzpatrick MJ (2010) A lover, not a fighter: Mating causes male crickets to lose fights. Behav Ecol Sociobiol 64:1971-1979

Kasumovic MM, Andrade MCB (2006) Male development tracks rapidly shifting sexual versus natural selection pressures. Curr Biol 16:R242-243

Kasumovic MM, Brooks R (2011) It's all who you know: The evolution of socially-cued anticipatory plasticity as a mating strategy. Q Rev Biol 86:181-197

Kasumovic MM, Elias DO, Punzalan D, Andrade MCB, Mason AC (2009) Experience affects the outcome of agonistic contests without affecting the selective advantage of size. Anim Behav 77:1533-1538

Kasumovic MM, Elias DO, Sivalinghem S, Mason AC, Andrade MCB (2010) Examination of prior contest experience and the retention of winner and loser effects Behav Ecol 21:404-409

Kasumovic MM, Hall MD, Brooks R (2012a) The juvenile social environment introduces variation in the choice and expression of sexually selected traits. Ecology and Evolution 2:1036-1047

Kasumovic MM, Hall MD, Try H, Brooks R (2011) The importance of listening: allocation shifts in response to the juvenile acoustic environment. J Evol Biol 24:1325-1334

Kasumovic MM, Hall MD, Try H, Brooks R (2012b) Socially cued developmental plasticity affects condition-dependent trait expression. Behav Ecol 24:429-434 
366

367

368

369

370

371

372

373

374

375

376

377

378

379

380

381

382

383

384

385

386

387

388

389

390

391

392

393

394

395

396

397

398

399

400

401

402

403

404

405

406

407

408

409

410

411

412

413

414

415

416
Kotiaho JS, Alatalo RV, Mappes J, Parri S (1999) Sexual signalling and viability in a wolf spider (Hygolycosa rubrofasciata): measurements under laboratory and field conditions. Behavioral Ecology and Sociobiology 46:123-128

Lailvaux SP, Hall MD, Brooks R (2010) Performance is no proxy for genetic quality: Trade-offs between locomotion, attractiveness, and life history in crickets. Ecology 91:1530-1537

Lailvaux SP, Kasumovic MM (2011) Defining individual quality over lifetimes and selective contexts. Proceedings B: Biological Sciences 278:321-328

Lailvaux SP, Zajitschek F, Dessman J, Brooks R (2011) Differential aging of bite and jump performance in virgin and mated teleogryllus commodus crickets. Evolution 65:3138-3147

Loher W (1974) Circadian control of spermatophore formation in the cricket Telleogryllus commodus. J Insect Physiol 20:1155-1172

Lomborg JP, Toft S (2009) Nutritional enrichment increases courtship intensity and improves mating success in male spiders. Behav Ecol 20:700-708

Papadopoulos NT, Katsoyannos BI, Kouloussis NA, Carey JR, Müller H, Zhang Y (2004) High sexual signalling rates of young individuals predict extended life span in male Mediterranean fruit flies. Oecologia 138:127-134

Pariser EC, Mariette MM, Griffith SC (2010) Artificial ornaments manipulate intrinsic male quality in wild-caught zebra finches (Taeniopygia guttata). Behav Ecol 21:264-269

Pinheiro J, Bates D, DebRoy S, Sarkar D (2009) nlme: Linear and nonlinear mixed effects models. R package version 3:1-96

Qvarnström A (1997) Experimentally increased badge size increases male competition and reduces male parental care in the collared flycatcher. Proceedings B: Biological Sciences 264:1225-1231

Rodd FH, Reznick DN, Sokolowski MB (1997) Phenotypic plasticity in the life history traits of guppies: Responses to social environment. Ecology 78:419-433

Rodriguez-Munoz R, Bretman A, Slate J, Walling CA, Tregenza T (2010) Natural and sexual selection in a wild insect population. Science 328:1269-1272

Rowe L, Houle D (1996) The lek paradox and the capture of genetic variance by condition dependent traits. Proc Roy Soc B 263:1415-1421

Royle NJ, Pike TW (2010) Social feedback and attractiveness in zebra finches. Behav Ecol Sociobiol 64:2015-2020

Stolz JA, Andrade MCB, Kasumovic MM (2012) Plasticity in metabolic rates reinforces morphological plasticity in response to social cues of sexual selection. J Insect Physiol 58:985-990

Tibbetts EA, Dale J (2004) A socially enforced signal of quality in a paper wasp. Nature 432:218-222

Walling CA, Royle NJ, Metcalfe NB, Linstrom J (2007) Green swordtails alter their age of maturity in response to the population level of male ornamentation. Biol Lett 3:144-146

Wood S (2009) gamm4: Generalized additive mixed models using mgcv and Ime4. In $\mathrm{R}$ package version 00-2 http://CRANR-projectorg/package=gamm4

Zajitschek F, Brassil CE, Bonduriansky R, Brooks RC (2009) Sex effects on life span and senescence in the wild when dates of birth and death are unknown. Ecology 90:1698-1707

Zajitschek F, Lailvaux SP, Dessmann J, Brooks R (2012) Diet, sex and death in field crickets. Ecology and Evolution 2:1627-1636

Zuk M, Kolluru GR (1998) Exploitation of sexual signals by predators and parasitoids. Quarterly Review of Biology 73:415-438 


\section{Table 1 (on next page)}

Table 1

The results of the model examining differences the effect of early mating on age-specific calling effort. 
PeerJ Reviewing Manuscript

\begin{tabular}{lrrr}
\hline Factor & \multicolumn{1}{c}{ d.f. } & F-value & $p$-value \\
\hline & 1, & & \\
Age & 1198 & 23.31 & $<0.0001$ \\
& 1, & & \\
& 1200 & 7.86 & 0.005 \\
Age $^{2}$ & 1,99 & 0.01 & 0.74 \\
Early mating & 1,99 & 0.18 & 0.67 \\
Late Mating & 1, & & \\
Early mating $\times$ & 1, & & \\
Age & 1198 & 2.29 & 0.13 \\
Late mating $\times$ & 1, & & \\
Age & 1198 & 0.11 & 0.74 \\
Early mating $\times$ & 1, & & \\
Age & 1198 & 5.54 & 0.019 \\
Late mating $\times^{2}$ & 1, & & \\
Age $^{2}$ & 1198 & 1.23 & 0.27 \\
\hline
\end{tabular}




\section{Table 2 (on next page)}

Model regression coefficients

Table 2: The regression coefficients of the linear (age) and quadratic (age2) terms of age for the individuals denied and granted their first mating opportunity. 


\section{PeerJ Reviewing Manuscript}

\begin{tabular}{lccc}
\hline & Early mating & Estimate & $p-$ \\
& & $\pm \mathrm{SE}$ & value \\
\hline \multirow{3}{*}{ Linear } & Denied & -8.46 & 0.006 \\
& & \pm 0.53 & \\
& Granted & - & $<0.00$ \\
& & $15.90 \pm 0.7$ & 01 \\
Quadrat & Denied & - & 0.49 \\
ic & & $2.10 \pm 1.83$ & \\
& Granted & - & $<0.00$ \\
& & $13.43 \pm 7.0$ & 01 \\
& & 7 & 01
\end{tabular}


Figure 1

Figure 1

The age-specific calling curves of males that failed and succeeded in their early mating. The dotted lines are $95 \%$ confidence intervals.
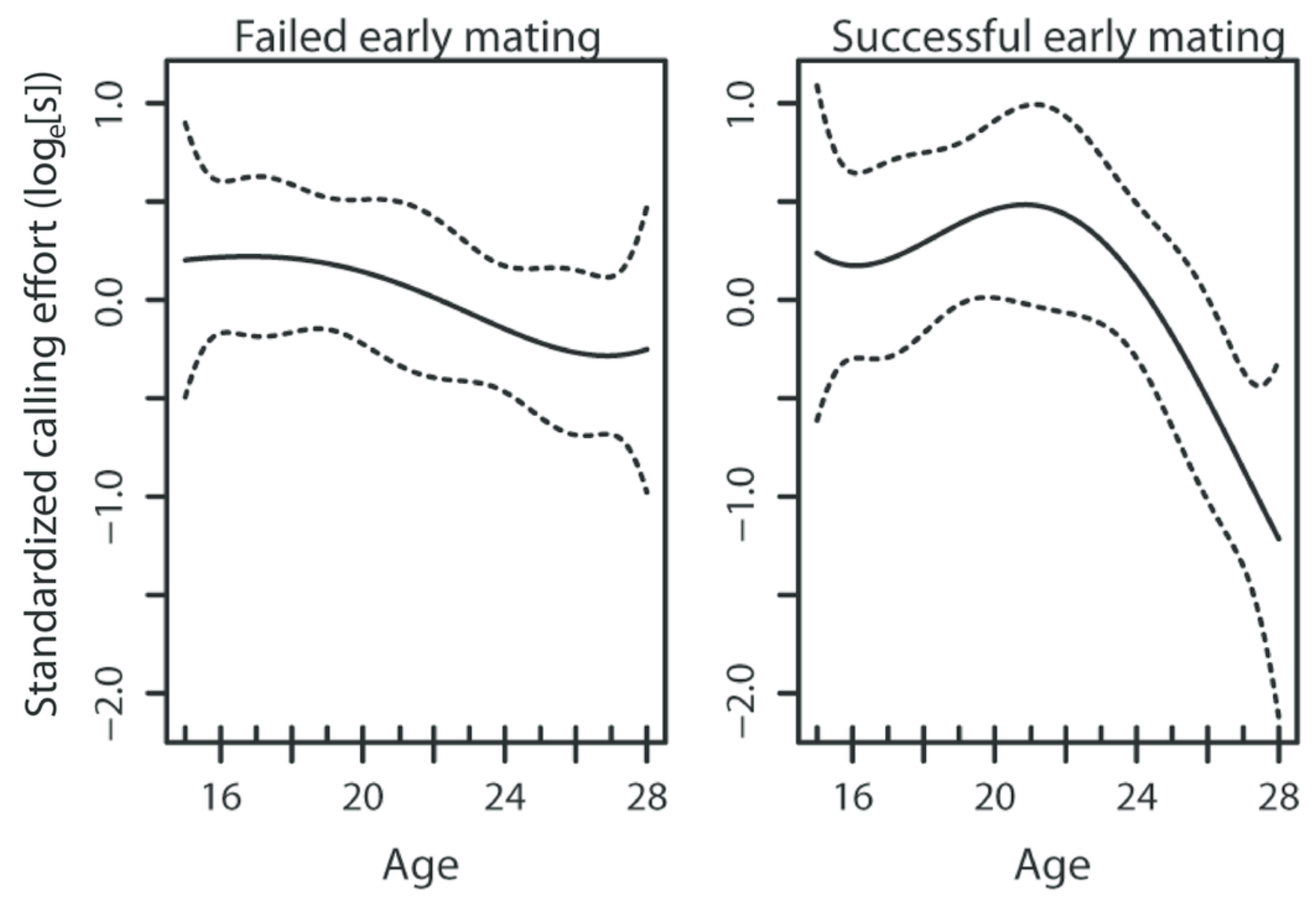